\title{
Avaliação funcional do reparo artroscópico das lesões completas do manguito rotador associado a acromioplastia
}

\author{
Functional evaluation of arthroscopic repairs of \\ complete rotator cuff tears associated to acromioplasty
}

Marco antonio de Castro Veado', Ildeu Afonso de Almeida Filho², Rafael Gonçalves Duarte ${ }^{3}$, Igor Leitão 4

\section{RESUMO}

Objetivo: Realizar avaliação funcional dos pacientes portadores de lesão completa do MR que foram submetidos ao reparo artroscópico associados à acromioplastia. Métodos: Trata-se de um estudo retrospectivo realizado com pacientes submetidos a tratamento cirúrgico artroscópico para reparo da lesão do MR. Foram incluídos os pacientes operados entre junho/2000 e outubro/2004 nos Hospitais Mater Dei e Felício Rocho, em Belo Horizonte. Dos 102 pacientes submetidos à reconstrução, 11 foram retirados por não cumprirem os critérios de inclusão, sendo o número final igual a 91 ombros em 91 pacientes. A avaliação funcional foi realizada pela escala $U C L A$ (Universidade da Califórnia em Los Angeles) e pelo teste Simples. Resultados: Na avaliação pós-operatória em 35 pacientes, os resul-

* Trabalho realizado no Hospital Mater Dei e Hospital Felicio Rocho - Belo Horizonte (MG), Brasil.

1. Médico Ortopedista do Serviço de Ortopedia do Hospital Mater Dei - Belo Horizonte (MG), Brasil.

2. Coordenador do Grupo de Ombro e Cotovelo do Hospital Felício Rocho - Belo Horizonte (MG), Brasil.

3. Médico Ortopedista formado no Hospital Mater Dei - Belo Horizonte (MG), Brasil.

4. Médico Ortopedista, Especialista em Cirurgia do Ombro e Cotovelo - Juiz de Fora (MG), Brasil.

Endereço para correspondência: Av. Celso Porfírio Machado, 104, Bairro Belvedere - 30320-400 - Belo Horizonte (MG), Brasil.

Recebido: 22.2.08. Autor para revisão: 10.4.08. Reapresentação: 12. 10.08. Aprovado: 27.11.08.

Copyright RBO2008 tados foram considerados excelentes $(38,4 \%)$; em 47, bons (51,6\%); seis, regulares $(6,6 \%)$; e três, ruins $(3,3 \%)$. Desse modo, $82(90,1 \%)$ pacientes foram avaliados como tendo obtido resultado bom ou excelente e nove como regulares ou ruins, sendo estes insatisfatórios. Conclusão: $\mathrm{O}$ reparo artroscópico do manguito rotador, associado a acromioplastia, apresentou resultado funcional excelente ou bom, na maioria dos pacientes, quando avaliados pelo método funcional da UCLA.

Descritores - Bainha rotadora/lesões; Bainha rotadora/cirurgia; Artroscopia/métodos; Procedimentos cirúrgicos reconstrutivos; Estudos de avaliação; Estudos retrospectivos

\section{ABSTRACT}

Objective: To perform a function evaluation of patients with complete rotator cuff tears that were submitted to arthroscopic repair associated to acromioplasty. Methods: This is a retrospective study made with patients submitted to arthroscopic surgical treatment to repair rotator cuff tears. Patients included were operated on from June 2000 to October 2004 at the Mater Dei and Felício Rocho Hospitals, in Belo Horizonte. Of the 102 patients submitted to reconstruction, 11 were removed from the study because they did not meet the inclusion criteria, and so the final number of the series was 91 shoulders of 91 patients. The functional evaluation scale was the UCLA scale and the simple test was performed. Results: In the post-operative evaluation of 35 
patients, results were considered excellent (38.4\%), 47 were considered good (51.6\%), six, regular, (6.6\%), and three poor results (3.3\%). 82 (90.1\%) patients therefore were evaluated as good or excellent results, and nine as regular or poor results, which were the unsatisfactory group in the series. Conclusion: The arthroscopic repair of rotator cuff, associated to acromioplasty, presented an excellent or good outcome for most patients when evaluated according to the UCLA functional method.

Keywords - Rotator cuff/injuries; Rotator cuff/surgery; Arthroscopy/methods; Reconstructive surgical procedures; Evaluation studies; Retrospective studies

\section{INTRODUÇÃO}

As lesões do manguito rotador (MR) são motivo freqüente de consultas médicas dentro do diagnóstico diferencial do ombro doloroso ${ }^{(1)}$.

Neer definiu a relação entre a morfologia do acrômio e a doença do MR, sugerindo a acromioplastia aberta como método de tratamento do impacto subacromial $^{(2)}$. Ellman introduziu a acromioplastia artroscópica como abordagem cirúrgica menos agressiva(3). Porém, nem todas as lesões do MR ocorrem como consequiência do impacto subacromial ou de um episódio traumático. Muitas lesões ocorrem por envelhecimento biológico ${ }^{(4)}$. É comum encontrarmos lesões assintomáticas em pacientes idosos, principalmente após os $70 \operatorname{anos}^{(5)}$.

Os resultados obtidos com o reparo artroscópico da lesão do MR têm-se mostrado satisfatórios ${ }^{(6-9)}$. A técnica vem sendo cada vez mais usada nos últimos anos. Em 1998, aproximadamente 5\% dos cirurgiões de ombro utilizavam o método artroscópico para o reparo da lesão do manguito rotador, sendo este valor de $24 \%$ em 2003 e $62 \%$ em $2005^{(10)}$. Nota-se que a técnica tem uma curva de aprendizado longa ${ }^{(10-11)}$. Millstein et al a consideram como padrão ouro para o reparo do MR e abordagem subacromial ${ }^{(11)}$.

A comparação com outros tipos de técnicas, como o reparo aberto clássico e a miniincisão associada a acromioplastia artroscópica (híbrida) ainda é válida e deve ser valorizada ${ }^{(12-14)}$. Esta última representa um período de transição na curva de aprendizado para a reconstrução totalmente artroscópica ${ }^{(15)}$. As abordagens abertas apresentam incidência maior de complicações, como agressão ao músculo deltóide, infecção, artrofibrose, cicatriz hipertrófica e dor pós-operatória ${ }^{(7,15-16)}$.

Outros pontos de discussão referem-se ao tipo de sutura no reparo do $\mathrm{MR}^{(17-18)}$, às vantagens e indicações de acromioplastia ${ }^{(19)}$ e aos riscos de injúrias relacionadas aos portais artroscópicos ${ }^{(20)}$.

O objetivo deste estudo é realizar uma avaliação funcional dos pacientes portadores de lesão completa do MR que foram submetidos ao reparo artroscópico associado a acromioplastia.

\section{MÉTODOS}

Trata-se de estudo retrospectivo realizado com pacientes submetidos a tratamento cirúrgico artroscópico para reparo da lesão do MR. A avaliação incluiu pacientes operados no período de junho/2000 a outubro/2004 nos Serviços de Ortopedia dos Hospitais Mater Dei e Felício Rocho. Dos 102 pacientes submetidos à reconstrução, 11 foram retirados por não cumprirem os critérios de inclusão, sendo o número final de 91 ombros em 91 pacientes. Dos pacientes, 59 $(64,8 \%)$ eram do sexo feminino e $32(35,2 \%)$, do masculino; $72(79,1 \%)$ tiveram o lado direito acometido e 19 (20,9\%), o esquerdo. O lado direito foi o dominante em 86 casos $(94,5 \%)$ e o esquerdo, em cinco $(5,5 \%)$. O tamanho da lesão foi considerado pequeno em 31 casos $(34,1 \%)$, médio em $46(50,5 \%)$ e grande em 14 $(15,4 \%)$ (gráfico 1). A média de idade foi de 62,1 anos

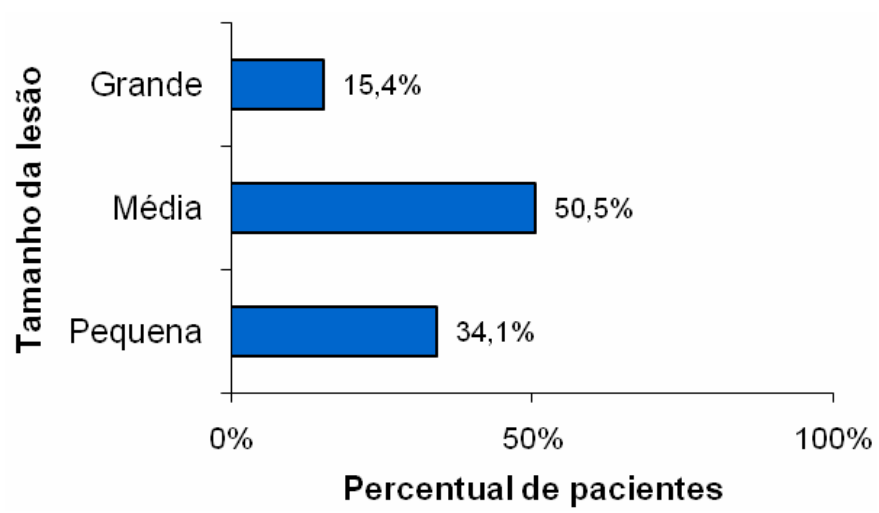

Gráfico 1 - Percentagem dos pacientes em relação ao tamanho das lesões do manguito rotador 


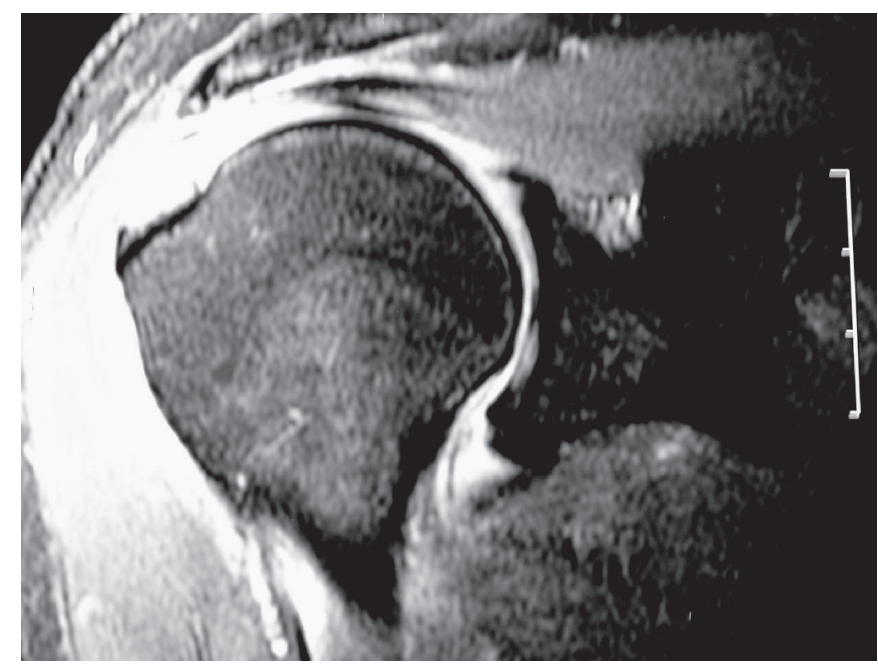

Figura 1 - Imagem coronal T2 de ressonância magnética do ombro mostrando lesão completa do tendão supra-espinhal

(42 a 81 anos). O acompanhamento médio foi 23,7 meses (12 a 60 meses). Utilizamos a seguinte classificação para definir o tamanho das lesões - pequenas: $<1 \mathrm{~cm}$; médias: 1 a $3 \mathrm{~cm}$; grandes: 3 a $5 \mathrm{~cm}$; e maciças: $>5 \mathrm{~cm}^{(21-22)}$.

Os critérios de inclusão neste estudo foram: lesões completas do MR, acompanhamento maior do que 12 meses e tempo de tratamento conservador mínimo de três meses, exceto nas lesões traumáticas agudas. $\mathrm{O}$ insucesso do tratamento conservador foi determinante para indicação cirúrgica.

Os critérios de exclusão englobaram: lesões parciais, lesões maciças, doenças associadas no ombro, como osteoartrose glenoumeral, lesões SLAP ou lesões do bíceps, pacientes envolvidos em questões trabalhistas, reoperações e pacientes psiquiátricos. Pacientes que apresentaram lesões associadas tiveram as mesmas tratadas durante o procedimento cirúrgico, sendo, porém excluídos deste estudo.

Todos os pacientes foram submetidos a acromioplastia artroscópica no mesmo ato operatório, geralmente realizada após o reparo do MR.

A avaliação retrospectiva foi realizada por dois observadores independentes em consultas de ambulatório. Comparamos os dados do prontuário do pré-operatório com os resultados do pós-operatório. Dentre os dados pré-operatórios disponíveis destaca-se a gra-

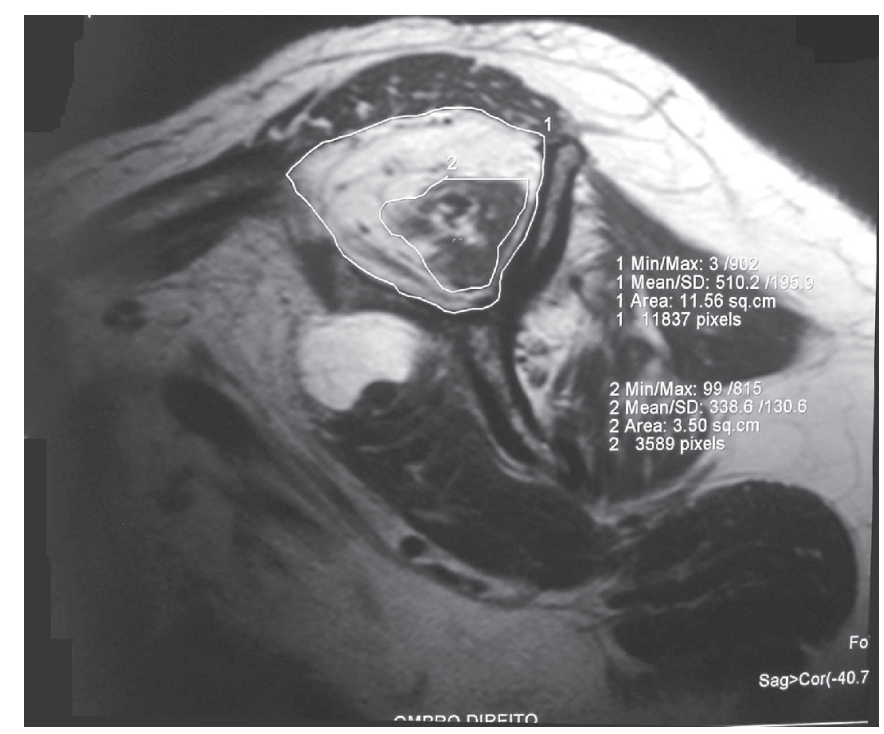

Figura 2 - Imagem sagital T1 de ressonância magnética do ombro mostrando atrofia do músculo supra-espinhal e infiltração gordurosa

duação funcional da UCLA (Universidade da Califórnia em Los Angeles) pré-operatória, feita de rotina pelos cirurgiões em seus pacientes, bem como dados obtidos de exames de ressonância magnética (figura 1), como o tamanho da lesão, presença de atrofias e infiltração gordurosa dos músculos do MR (figura 2). $\mathrm{Na}$ avaliação pós-operatória graduamos pela escala funcional da UCLA e utilizamos o teste funcional (Simple Shoulder Test), o qual não foi disponível no préoperatório.

A avaliação funcional da UCLA, previamente usada para artroplastias, é hoje constantemente utilizada para avaliar outras intervenções no ombro, inclusive as lesões do MR. Os parâmetros são: dor (10 pontos), movimento (10 pontos), função (10 pontos) e satisfação do paciente (5 pontos). Graduação menor ou igual a 20 indica resultado ruim; de 21 a 28, regular; de 29 a 33 bom; e 34 a 35 , excelente.

O Simple Shoulder Test constitui-se de 12 perguntas diretas, de resposta sim ou não. É também usado para avaliar a função no ombro.

Os testes estatísticos usados foram: coeficiente de correlação de Spearman, teste exato de Fisher, teste não paramétrico de Kruskal-Wallis e teste não paramétrico Wilcoxon Signed Rank. 


\section{Técnica cirúrgica}

Os pacientes foram colocados na posição em "cadeira de praia" ou em decúbito lateral, conforme preferência de cada cirurgião. O bloqueio do plexo braquial associado à anestesia geral com intubação orotraqueal foi utilizado em todos os pacientes. Na grande maioria das vezes, os portais usados foram o anterior, lateral e posterior.

Após a introdução do artroscópio, um inventário completo da articulação glenoumeral constituiu uma rotina de avaliação. Procuraram-se lesões associadas como as do tendão bicipital, lesões SLAP, além da presença de corpos livres articulares, sendo tratadas quando necessário.

Em seguida, buscou-se inspecionar o espaço subacromial para avaliar os tendões do MR. Para isso, muitas vezes é imperativo proceder a uma bursectomia ampla para boa visualização da lesão e avaliação de suas características, como tamanho, retração e qualidade tecidual. Nas lesões mais retraídas os tendões foram liberados pelo seu lado bursal, para evitar tensão na sutura. Eventualmente, foi necessário também fazer uma capsulotomia interna para melhor liberação dos tendões. O desbridamento econômico das bordas da lesão, bem como o preparo da zona de reinserção do MR em posição justarticular, foi sempre realizado. Os tendões foram reinseridos usando-se fios inabsorvíveis e âncoras de titânio de 5,0mm, separadas entre si por uma distancia média de $1 \mathrm{~cm}$ (figura 3 ). Um recurso usado com frequiência para diminuir o tamanho da lesão foi a sutura látero-lateral, nas lesões em forma de "U".

A acromioplastia foi um procedimento realizado nesta amostragem em todos pacientes, sendo feita geralmente após o reparo do MR. Eventualmente, quando na presença de um acrômio curvo ou ganchoso, resultando em um espaço subacromial muito apertado, demos preferência a fazer a acromioplastia como procedimento inicial.

O pós-operatório incluiu a proteção do reparo com o uso de tipóia tipo Velpeau por seis semanas. Na primeira revisão tem-se como hábito fazer radiografias para avaliação do posicionamento das âncoras. Exercícios passivos de elevação anterior são permitidos

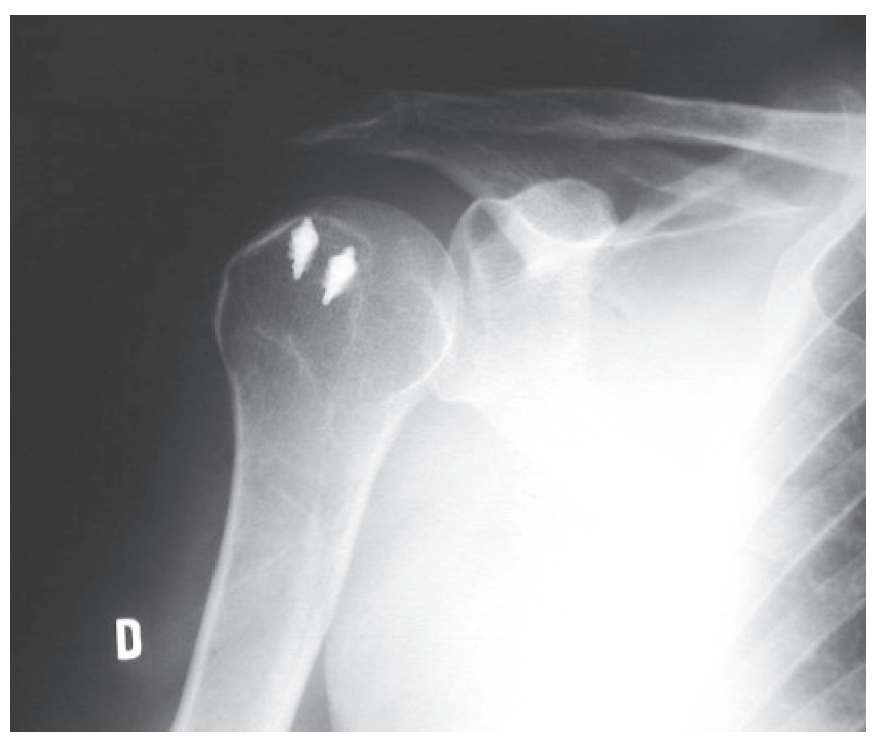

Figura 3 - Radiografia ântero-posterior do ombro: duas âncoras inseridas no tubérculo maior para reparo do manguito rotador.

assim que o paciente puder suportar a dor. Os exercícios pendulares são iniciados após a terceira semana. Após a remoção da tipóia, os pacientes foram encaminhados para a clínica de reabilitação para continuar com o ganho dos movimentos e recuperação da força muscular.

\section{RESULTADOS}

A graduação da UCLA pré-operatória média foi de 15,15 (8 a 23 pontos); a UCLA pós, de 32,45 (18 a 35 pontos); e o teste simples, 11,25 (5 a 12 pontos) (tabela 1).

Na avaliação pós-operatória obtivemos resultados em 35 pacientes considerados excelentes $(38,4 \%)$; em 47 , bons $(51,6 \%)$; em seis, regulares $(6,6 \%)$; e em três, ruins $(3,3 \%)$. Desse modo, em $82(90,1 \%)$ pacientes, os resultados foram avaliados como bons ou excelentes, ou seja, satisfatórios, e, em nove pacientes, ruins ou regulares, sendo considerados como insatisfatórios.

A média de idade variou de 68,6 anos nos resultados ruins, 67,6 nos regulares, 63,3 nos bons e 59 nos excelentes.

Os resultados obtidos referentes aos valores médios da avaliação funcional da UCLA e do Simple Shoulder Test nos diferentes tamanhos das lesões estão na tabela 2. 
TABELA 1

Avaliação funcional pré e pós-operatória e idade

\begin{tabular}{lccccccc}
\hline \multicolumn{1}{c}{ Variáveis } & $\mathbf{n}$ & Média & Mediana & $\begin{array}{l}\text { Desvio- } \\
\text { padrão }\end{array}$ & Mínimo & Máximo & Valor $\mathbf{p}$ \\
\hline Idade (em anos) & 91 & 62,13 & 63 & 8,35 & 42 & 81 & \\
\hline UCLA pré & 91 & 15,15 & 16 & 3,69 & 8 & 23 & $\mathbf{0}, 0000 *$ \\
UCLA pós & 91 & 32,45 & 33 & 3,53 & 18 & 35 & 12 \\
\hline Teste Simples & 91 & 11,25 & 12 & 1,40 & 5 & \\
\hline
\end{tabular}

TABELA 2

Avaliação funcional pré e pós-operatória e tamanho da lesão

\begin{tabular}{|c|c|c|c|c|c|c|c|c|}
\hline Variáveis & Tamanho & $n$ & Média & Mediana & $\begin{array}{l}\text { Desvio } \\
\text { padrão }\end{array}$ & Mínimo & Máximo & Valor $p$ \\
\hline & $\mathrm{P}$ & 31 & 15,9032 & 17 & 2,856 & 10 & 23 & \\
\hline \multirow[t]{3}{*}{ UCLA pré } & $\mathrm{M}$ & 46 & 14,6739 & 15 & 3,7774 & 8 & 23 & 0,2995 \\
\hline & G & 14 & 15,0714 & 15,5 & 4,8748 & 8 & 23 & \\
\hline & $\mathrm{P}$ & 31 & 33,2903 & 33 & 2,0362 & 27 & 35 & \\
\hline \multirow[t]{3}{*}{ UCLA pós } & $\mathrm{M}$ & 46 & 32,1522 & 33 & 3,7474 & 18 & 35 & 0,3776 \\
\hline & G & 14 & 31,5714 & 34 & 5,034 & 20 & 35 & \\
\hline & $P$ & 31 & 11,6774 & 12 & 0,7018 & 9 & 12 & \\
\hline \multirow{2}{*}{ Teste Simples } & $\mathrm{M}$ & 46 & 11,1087 & 12 & 1,4792 & 5 & 12 & 0,0712 \\
\hline & $\mathrm{G}$ & 14 & 10,7857 & 12 & 2,0069 & 5 & 12 & \\
\hline
\end{tabular}

Dos seis pacientes com resultados regulares, conseguimos evidenciar possível causa em dois casos. Um deles não cumpriu o período de fisioterapia pós-operatória e o outro, com distúrbios do equilíbrio, sofria quedas freqüentes, com provável rerruptura do MR. Os quatro restantes cumpriram o programa pós-operatório, não sendo identificada nenhuma causa aparente.

Entre os avaliados com resultados ruins, podemos citar um que desenvolveu uma distrofia simpático-reflexa pós-operatória. Outro sofreu trauma no pós-operatório com rerruptura e o último, era portador de osteoartrose cervical associada a braquialgia, não se mostrou muito satisfeito pelo agravamento da dor no membro operado.

Pode-se dizer que existe uma diferença estatisticamente significante entre a avaliação funcional da UCLA pré-operatória e da pós-operatória (valor $\mathrm{p}<0,05$ ). Além disso, é possível dizer que a avaliação funcional da UCLA pós-operatória é significativamente maior do que a UCLA pré.
Na relação idade versus valor da UCLA pós-operatória e idade versus Simple Shoulder Test, podemos afirmar que há relação significativa e negativa (valor $\mathrm{p}<$ $0,05)$, conforme a tabela 3. Não é possível, porém, dizer que o tamanho da lesão ou o tempo de seguimento influenciaram no resultado do valor da UCLA pós-operatória ou do Simple Shoulder Test".

\section{TABELA 3}

Avaliação funcional pós-operatória, idade e seguimento

\begin{tabular}{lcc}
\hline \multicolumn{1}{c}{ Variáveis } & $\begin{array}{c}\text { Coeficiente de } \\
\text { correlação de } \\
\text { Spearman }\end{array}$ & Valor $\mathbf{p}$ \\
\hline Seguimento x UCLA pós & 0,116 & 0,272 \\
Idade x UCLA pós & $-0,266$ & $\mathbf{0 , 0 1 1}$ \\
Seguimento x Teste simples & 0,017 & 0,872 \\
Idade x Teste simples & $-0,308$ & $\mathbf{0 , 0 0 3}$ \\
\hline
\end{tabular}

\section{DISCUSSÃO}

Habitualmente o tratamento conservador das lesões do MR deve ser recomendado antes de se indicar o tra- 
tamento cirúrgico ${ }^{(7-9)}$. O período médio sugerido é aproximadamente de três a seis meses. Gartsman considera que o tratamento cirúrgico deve ser pensado quando a dor piora após seis meses ou não melhora após um ano de tratamento conservador ${ }^{(16)}$. Na doença do MR observamos considerável percentagem de lesões assintomáticas, principalmente em idosos ${ }^{(5)}$. O cirurgião deve levar em conta ainda as características da lesão, os sintomas, a demanda do paciente, bem como a confiabilidade de adesão ao período de reabilitação ${ }^{(11)}$. Por essas razões, nossa conduta inicial tem sido mais conservadora, principalmente nos idosos e sedentários. Em pacientes jovens, esportistas ou trabalhadores, tendemos a encurtar esse tempo. Nas rupturas traumáticas agudas dos mais jovens achamos mais conveniente reparar os tendões mais precocemente, a não ser que o paciente se apresente com um ombro rígido ${ }^{(21)}$.

Outra consideração importante a se fazer é em relação à atrofia e à infiltração gordurosa dos músculos do manguito rotador. Sabe-se que a sua instalação pode ocorrer precocemente em alguns pacientes ${ }^{(2)}$. Desse modo, ao protelarmos o ato cirúrgico estaríamos perdendo um tempo precioso, operando mais tardiamente um tendão cujo músculo adjacente apresenta pouca capacidade de gerar força e pequeno potencial de reversão do quadro de atrofia e infiltração gordurosa ${ }^{(23)}$.

Checchia et al e Gartsman et al relataram em seus trabalhos que não houve influência da idade na época da cirurgia, tempo de seguimento e tamanho da lesão no resultado funcional pós-operatório ${ }^{(7,24)}$. Em nosso estudo, a idade foi fator preponderante no resultado, influenciando negativamente. Já o acompanhamento e o tamanho da lesão não influenciaram estatisticamente o resultado da UCLA ou Simple Shoulder Test. Na comparação entre UCLA pré e UCLA pós houve diferença estatisticamente significante e positiva. O Teste Simples indicou-nos apenas um resultado instantâneo pós-operatório, não permitindo comparação pré e pósoperatória porque os valores pré-operatórios não eram disponíveis.

A abordagem artroscópica do MR tem inúmeras vantagens, que incluem pequena incisão, menor índice de infecção, não agressão da inserção do músculo deltói- de, menor dor pós-operatória e recuperação mais rápida. As desvantagens relacionam-se a maior custo do procedimento e à necessidade de curva de aprendizado mais longa. Recentemente, avanços podem ser observados no método e devem-se principalmente às melhorias nos instrumentais e à experiência acumulada dos cirurgiões ${ }^{(9)}$.

Burkhart et al classificaram as lesões completas do MR em dois tipos, de acordo com seu formato ${ }^{(10)}$. A forma em crescente apresenta-se como grande lesão, sendo geralmente pouco retraída, o que permite sua reinserção diretamente no osso. A forma em "U" é mais retraída e exige sutura tendão-tendão antes de reinserção óssea final. Em nossa experiência esse foi um recurso bastante utilizado para diminuir o tamanho da lesão, tornando a fixação mais simples e mais econômica.

Snyder et al publicaram trabalho relatando $87 \%$ de bons e excelentes resultados no reparo do MR por via artroscópica e Checchia et al, numa casuística de 141 pacientes, $93,7 \%{ }^{(6-7)}$. Wolf et al obtiveram $94 \%$ de resultados satisfatórios com o mesmo método, Burkhart et al relataram $95 \%$ e Godinho, $92 \%{ }^{(8,25-26)}$. Em nosso estudo obtivemos $90,1 \%$ de resultados satisfatórios, estando também de acordo com os de outros pesquisadores.

A comparação do reparo artroscópico com as técnicas abertas e semi-abertas ainda é foco de discussão. Miyazaki et al obtiveram $85,7 \%$ de resultados satisfatórios com acromioplastia artroscópica e reparo do MR por miniincisão ${ }^{(12)}$. Veado et $a l$, em uma casuística de 47 pacientes, publicaram resultados semelhantes com a mesma técnica ${ }^{(13)}$. A técnica aberta clássica apresenta maior índice de infecção, agressão ao músculo deltóide, artrofibrose, cicatriz hipertrófica e dor pós-operatória $^{(7,15-16)}$.

A realização ou não da acromioplastia ainda é tema controverso. Elman descreveu acromioplastia artroscópica, com resultados tão satisfatórios quanto por via aberta $^{(27)}$. Snyder et al acreditam que a descompressão subacromial deve ser feita se a lesão acomete os lados articular e bursal do tendão(28). Caso a lesão seja apenas na face articular, preconizam apenas o reparo do MR. Em estudo de lesões maciças, Scheibel et al reali- 
zaram o procedimento de descompressão subacromial reversa com preservação do arco coracoacromial, conseguindo melhorar a dor, ADM e a função em todos os 22 pacientes avaliados ${ }^{(29)}$. A presença de lesões irreparáveis com migração superior da cabeça umeral representa uma contra-indicação formal à acromioplastia. Uma das contra-indicações relativas seria a presença do os acromiale $^{(30)}$. Nesse estudo foi realizada acromioplastia em todos pacientes.

Há também na literatura descrições sobre as rerrupturas. Lo et al avaliaram 14 pacientes submetidos a revisão de falha no reparo MR. Identificaram, pela avaliação funcional da UCLA, nove de 14 resultados considerados satisfatórios ${ }^{(30)}$. Em nosso estudo houve um caso de rerruptura comprovada e outro de provável rerruptura em paciente com distúrbios de equilíbrio que sofria quedas freqüentes.

Lee et al publicaram estudo comparando a fixação de âncoras absorvíveis sem uso de sutura com âncoras padronizadas que utilizam sutura. Concluíram que as bioabsorvíveis, apesar de mais práticas, podem não conferir fixação suficiente ao reparo do MR em humanos, principalmente na reabilitação pós-operatória ${ }^{(18)}$. Em nossa amostragem, usamos somente âncoras de titânio de 5,0mm com fio de poliéster trançado de 2,0mm. Existem fios da mesma espessura e com maior resistência mecânica, porém, não foram utilizados neste estudo.

No que se refere aos portais artroscópicos, Lo et al sugeriram que dificilmente ocorrem danos neurológi$\cos ^{(30)}$. No portal anterior há o risco de lesão da veia cefálica, porém, com subseqüente pouca morbidade ${ }^{(20)}$. Não tivemos em nosso estudo casos de danos neurovasculares relacionados aos portais artroscópicos.

\section{CONCLUSÃO}

O reparo artroscópico do manguito rotador, associado à acromioplastia, apresenta resultados funcionais excelentes e bons, na maioria dos pacientes, quando avaliados pelo método funcional da UCLA.

\section{REFERÊNCIAS}

1. Andrade RP, Correa Filho MRC, Queiroz BC. Lesões do manguito rotador. Rev Bras Ortop. 2004;39(11/12):621-36.
2. Neer CS 2nd. Impingement lesions. Clin Orthop Relat Res. 1983;(173):70-7.

3. Ellman H. Diagnosis and treatment of incomplete rotator cuff tears. Clin Orthop Relat Res. 1990;(254):64-74. Review.

4. Nottage WM. Rotator cuff repair with or without acromioplasty. Arthroscopy. 2003;19 Suppl 1:229-32. Review.

5. Sher JS, Uribe JW, Posada A, Murphy BJ, Zlatkin MB. Abnormal findings on magnetic ressonance images of asymptomatic shoulders. J Bone Joint Surg Am. 1995;77(1): 10-5. Comment in: J Bone Joint Surg Am. 1996;78(4):633. J Bone Joint Surg Am. 1996;78(4):633-5.

6. Snyder SJ, Heath DD. Arthroscopic repair of rotator cuff tears with miniature suture screw anchors and permanent mattress sutures. Arthroscopy. 1994;10:345.

7. Checchia SL, Doneux SP, Miyazaki AN, Fregoneze M, Silva LA, Ishi M, et al. Avaliação dos resultados obtidos na reparação artroscópica das lesões do manguito rotador. Rev Bras Ortop. 2005;40(5):229-38.

8. Wolf EM, Pennington WT, Agrawal V. Arthroscopic rotator cuff repair: 4- to 10-year results. Arthroscopy. 2004;20(1):512.

9. Park JY, Chung KT, Yoo MJ. A serial comparison of arthroscopic repairs for partial and- full-thickness rotator cuff tears. Arthroscopy. 2004;20(7):705-11.

10. Burkhart SS, Lo IK. Arthroscopic rotator cuff repair. J Am Acad Orthorp Surg. 2006;14(6):333-46.

11. Millstein ES, Snyder SJ. Arthroscopic management or partial, full-thickness, and complex rotator cuff tears: indications, techniques, and complications. Arthroscopy. 2003;19 Suppl 1: 189-99. Review.

12. Miyazaki AN, Doneaux SP, Saito RY, Kussakawa D, Checchia SL. Acromioplastia artroscópica e reparo das lesões do manguito rotador por “miniincisão”. Rev Bras Ortop. 1999;34(7):41520.

13. Veado MAC, Higino GC, Almeida Filho IA. Descompressão subacromial artroscópica com reparo do manguito rotador pela miniincisão. Rev Bras Ortop. 2004;39(3):102-11.

14. Kim SH, Ha KI, Park JH, Kang JS, Oh SK, Oh I. Arthroscopic versus mini-open salvage repair of the rotator cuff tear: outcome analysis at 2 to 6 years' follow-up. Arthroscopy. 2003; 19(7):746-54.

15. Godinho GG, Souza JMG, Bicalho LA. Reparo das rupturas do manguito rotador do ombro pela video artroscopia cirúrgica: técnica. Rev Bras Ortop. 1996;31(4):284-8.

16. Gartsman GM. Arthoscopic management of rotator cuff disease. J Am Acad Orthop Surg. 1998;6(4):259-66.

17. Dejong ES, DeBerardino TM, Brooks DE, Judson K. In vivo comparison of a metal versus a biodegradable suture anchor. Arthroscopy. 2004;20(5):511-6.

18. Lee S, Mahar A, Bynum K, Pedowitz R. Biomechanical comparison of bioabsorbable sutureless screw anchor versus 
suture anchor fixation for rotator cuff repair. Arthroscopy. 2005;21(1):43-7.

19. Nicoletti SJ, Carrera EF, Archetti Neto N, Viveiros MEN, Manetta Filho E. Tratamento artroscópico da síndrome do pinçamento subacromial, com e sem acromioplastia: resultados após seguimento médio de 45 meses. Rev Bras Ortop. 1998;33(5): $333-7$.

20. Lo IK, Lind CC, Burkhart SS. Glenohumeral arthroscopy portals established using an outside-in technique: neurovascular anatomy at risk. Arthroscopy. 2004;20(6):596602.

21. Fukuda H. Partial-thickness rotator cuff tears: a modern view on Codman's classic. J Shoulder Elbow Surg. 2000;9(2):163-8. Review.

22. Romeo AA, Mazzocca A, Hang DW, Shott S, Bach BR Jr. Shoulder scoring scales for the evaluation of rotator cuff repair. Clin Orthop Relat Res. 2004;(427):107-14.

23. Goutallier D, Postel JM, Bernageu J, Lavau L, Voisin MC. Fatty muscle degeneration in cuff ruptures. Pre- and postoperative evaluation by CT scan. Clin Orthop Relat Res. 1994;(304):7883.
24. Gartsman GM, Khan M, Hammerman SM. Arthroscopic repair of full-thickness tears of the rotator cuff. J Bone Joint Surg Am. 1998;80(6):832-40.

25. Burkhart SS, Danaceau SM, Pearce CE Jr. Arthroscopic rotator cuff repair: Analysis of results by tear size and by repair technique-margin convergence versus direct tendon-to-bone repair. Arthroscopy. 2001;17(9):905-12.

26. Godinho GG. Reparação artroscópica do manguito rotador do ombro: avaliação funcional dos resultados de 87 pacientes [tese]. São Paulo: Universidade Federal de São Paulo. Escola Paulista de Medicina; 2002.

27. Ellman H. Arthroscopic subacromial decompression: analysis of one- to three-year results. Arthroscopy. 1987;3(3):173-81.

28. Snyder SJ, Pachelli AF, Del Pizzo W, Friedman MJ, Ferkel RD, Pattee G. Partial thickness rotator cuff tears: results of arthroscopic treatment. Arthroscopy. 1991;7(1):1-7.

29. Scheibel M, Lichtenberg S, Habermeyer P. Reversed arthroscopic subacromial decompression for massive rotator cuff tears. J Shoulder Ellbow Surg. 2004;13(3):272-8.

30. Lo IK, Burkhart SS. Arthroscopic revision of failed rotator cuff repairs: technique and results. Arthroscopy. 2004;20(3):25067.

\footnotetext{
Declaração de inexistência de conflitos de interesse: Declaramos inexistência de conflitos de interesses.
} 